\title{
Predicting High-Flow Spinal CSF Leaks in Spontaneous Intracranial Hypotension Using a Spinal MRI-Based Algorithm: Have Repeat CT Myelograms Been Reduced?
}

\author{
J.T. Verdoorn, P.H. Luetmer, C.M. Carr, J.I. Lane, D.T. Lehman, DJ.M. Morris, K.R. Thielen, J.T. Wald, and (DF.E. Diehn
}

\begin{abstract}
BACKGROUND AND PURPOSE: We adopted an imaging algorithm in 2011 in which extradural fluid on spinal MR imaging directs dynamic CT myelography. We assessed algorithm compliance and its effectiveness in reducing repeat or unnecessary dynamic CT myelograms.

MATERIALS AND METHODS: CT myelograms for CSF leaks from January 2011 to September 2014 were reviewed. Patients with iatrogenic leaks, traumatic brachial plexus injuries, or prior CT myelography within 2 years were excluded. Completion and results of spinal MR imaging, CT myelographic technique, and the need for repeat CT myelography or unnecessary dynamic CT myelograms were recorded.

RESULTS: The algorithm was followed in 102 (79\%) of 129 patients. No extradural fluid was detected in 75 (74\%), of whom 70 (93\%) had no leak, $4(5 \%)$ had a slow leak, and $1(1 \%)$ had a fast leak. Extradural fluid was detected in 27 (26\%): 24 (89\%) fast leaks, 1 (4\%) slow leak, and 2 (7\%) with no leaks. When the algorithm was followed, 1 (1\%) required repeat CT myelography and 3 (3\%) had unnecessary dynamic CT myelograms. The algorithm was breached in 27 (21\%) cases, including no pre-CT myelogram MR imaging in 11 (41\%), performing conventional CT myelography when extradural fluid was present in 13 (48\%), and performing dynamic CT myelography when extradural fluid was absent in $3(11 \%)$. Algorithm breaches resulted in 4 (15\%) repeat CT myelograms and 3 (12\%) unnecessary dynamic CT myelograms, both higher than with algorithm compliance.
\end{abstract}

CONCLUSIONS: Using spinal MR imaging to direct CT myelography resulted in significant reduction in repeat CT myelograms to localize fast leaks with minimal unnecessary dynamic CT myelograms.

ABBREVIATION: $\mathrm{CTM}=\mathrm{CT}$ myelogram

$\mathbf{E}^{\mathrm{x} x}$ xtradural fluid on spinal MR imaging has been reported to Epredict fast spinal CSF leaks for which the leak site may not be localized on conventional CT myelograms (CTMs). ${ }^{1}$ We adopted an imaging algorithm in January 2011 for the evaluation of patients with clinical suspicion of spinal CSF leak. The first step of this algorithm is to perform MR imaging of the entire spinal canal, and the results of the MR imaging are then used to guide the type of CTM initially performed. Specifically, if extradural fluid is present on MR imaging, dynamic CTM is performed. Our current technique used for dynamic CTM has been previously reported. ${ }^{2}$ If extradural fluid is not present, conventional CTM is performed.

The goal of adopting this algorithm was to attempt to reduce

Received March 15, 2015; accepted after revision May 21.

From the Department of Radiology, Mayo Clinic Rochester, Rochester, Minnesota. Paper previously presented at: Annual Meeting of the American Society of Neuroradiology and the Foundation of the ASNR Symposium; April 24-30, 2015; Chicago, Illinois.

Please address correspondence to Patrick H. Luetmer, MD, Department of Radiology, Mayo Clinic Rochester, 200 First St SW, Rochester, MN 55905; e-mail:

luetmer.patrick@mayo.edu

http://dx.doi.org/10.3174/ajnr.A4465 the number of repeat dynamic CTMs for leak localization in patients with fast spinal CSF leaks who initially underwent conventional CTM with the leak identified but not localizable. Averaged over the previous 8 years, repeat dynamic CTM for leak localization was performed in $21 \%$ of patients at our institution. ${ }^{1}$ Reducing repeat CTM is desirable for several reasons, including radiation reduction, cost savings, and fewer invasive procedures.

The purpose of this study was to retrospectively evaluate our compliance with the algorithm and determine its effectiveness in reducing repeat dynamic CTM performed for leak localization.

\section{MATERIALS AND METHODS}

After obtaining institutional review board approval, a retrospective review was performed of all patients referred to CTM for suspected spinal CSF leak between January 2011 and September 2014 , as determined by a radiology information system data base search. Referral to CTM was based on a working clinical diagnosis of spontaneous intracranial hypotension, typically with a history of orthostatic headache, as determined following evaluation by a headache neurologist. 
Patients with spinal CSF leaks secondary to an iatrogenic cause or traumatic brachial plexus injuries, as determined by review of radiology reports and the electronic medical record, were excluded. Patients with a prior CTM within 2 years, as determined by review of prior radiology examinations performed at our institution or an outside facility, were also excluded because the presence and rate of a CSF leak on the prior study could bias selection of CTM examination type.

The radiology report from each CTM was reviewed to determine the type of CTM performed. This was categorized as either conventional or dynamic. The report was also reviewed to determine whether a spinal CSF leak was present or absent. If a leak was present, it was categorized as either a fast or slow leak. A "fast leak" was defined as a leak that required the initial dynamic series of a dynamic CTM for leak localization. The initial dynamic series of a dynamic CTM includes up to 6 serial CT scans of the spine obtained during intrathecal contrast injection over the course of approximately 75 seconds, as previously described by our group. ${ }^{2}$ All other leaks were defined as slow leaks.

Whether a spinal MR imaging examination was completed within the year preceding the CTM was recorded. The spinal MR imaging could have been from our institution or from an outside facility, if electronically available. If a spinal MR imaging examination was completed, the presence or absence of an extradural fluid collection on that examination was recorded on the basis of review of the radiology report. Each of these spinal MR imaging examinations had been interpreted by a staff neuroradiologist from our institution. If no report was available for a spinal MR imaging performed at an outside facility, the images were reviewed by a staff neuroradiologist from our institution to determine the presence or absence of an extradural fluid collection. The segments of the spine imaged on MR imaging were also recorded. These were divided into cervical, thoracic, lumbar, and each possible combination thereof. The number of days elapsed between the spinal MR imaging and the CTM was also noted. Any interventional treatment performed for spontaneous intracranial hypotension between the spinal MR imaging and the CTM was also recorded.

On the basis of whether spinal MR imaging was performed, the presence or absence of extradural fluid on MR imaging, the type of CTM performed, whether a spinal CSF leak was identified on the CTM, and whether that leak was fast or slow, the algorithm was analyzed for compliance and success as described below.

The number of patients in whom the algorithm was followed (ie, compliance) included all patients who had pre-CTM spinal MR imaging of any segments and who were appropriately triaged for dynamic CTM if extradural fluid was present on the MR imaging or conventional CTM if extradural fluid was not present.

Algorithm success was defined as patients in whom the algorithm was followed, did not require repeat imaging with a dynamic CTM for leak localization, and did not undergo unnecessary dynamic CTM for a slow or absent CSF leak. Algorithm failure was defined as patients in whom the algorithm was followed but either required repeat imaging with dynamic CTM for leak localization or underwent initial dynamic CTM for a slow or absent CSF leak.

The number of patients in whom the algorithm was not fol- lowed included all patients with one of the following breaches: patients without pre-CTM spinal MR imaging, patients inappropriately triaged for dynamic CTM if extradural fluid was not present on the MR imaging, or patients inappropriately triaged for conventional CTM if extradural fluid was present on the MR imaging.

The number of unnecessary dynamic CTMs for slow or absent CSF leaks and nonlocalized fast leaks on conventional CTM was recorded for cases in which the algorithm was breached and also for cases in which it was followed. These were compared by using a 2-tailed Fisher exact test.

\section{RESULTS}

\section{Study Population}

We identified 181 patients who were referred to CTM for a suspected spinal CSF leak. Of those, 52 (29\%) were excluded. Reasons for exclusion in these patients were the following: 37 (71\%) for prior CTM within 2 years, 12 (23\%) for iatrogenic leaks, and 3 (6\%) for leaks related to traumatic brachial plexus injuries. The remaining 129 patients were included in the study population.

In the 118 patients who underwent spinal MR imaging, the average number of days between spinal MR imaging and CTM was 50.4 (range, 0-351 days). Fifteen of 118 (13\%) patients underwent interventional treatment between the spinal MR imaging and CTM. Of these, 12 received nontargeted epidural blood patches, 2 received nontargeted epidural injections of blood and fibrin glue, and 1 underwent surgical dural repair.

\section{Algorithm Compliance}

The algorithm was followed in 102 (79\%) of 129 patients. In these patients, spinal MR imaging segments included the following: cervical/thoracic/lumbar in $82(80 \%)$, cervical/thoracic in $8(8 \%)$, cervical/lumbar in $2(2 \%)$, thoracic/lumbar in $2(2 \%)$, cervical in $2(2 \%)$, thoracic in $4(4 \%)$, and lumbar in $2(2 \%)$.

Of the 20 patients with only a portion of the spine imaged with MR imaging, 18 had no extradural fluid present. These patients all underwent conventional CTM with no leak identified in 16 (89\%) and slow leaks identified in $2(11 \%)$. In the 2 patients with slow leaks, the imaged portion of the spine on MR imaging included the suspected site of leak identified on CTM. The 2 patients with extradural fluid present were appropriately triaged to dynamic CTM with fast leaks identified.

In the 102 patients in whom the algorithm was followed, extradural fluid was present on spinal MR imaging in 27 (26\%) and absent in 75 (74\%). When extradural fluid was present, dynamic CTM was performed and demonstrated a fast leak in 24 (89\%) of 27 patients (Fig 1), a slow leak in 1 (4\%), and no identifiable leak in $2(7 \%)$. When extradural fluid was absent, conventional CTM was performed and demonstrated no identifiable leak in 70 (93\%) of 75 patients, a slow leak in $4(5 \%)$, and a fast leak that could not be localized in $1(1 \%)$.

\section{Algorithm Success}

When the algorithm was followed, algorithm success was present in 98 (96\%) of 102 patients, and algorithm failure, in 4 (4\%).

As for the failures, 3 (3\%) of 102 patients underwent unnecessary dynamic CTM for a slow or absent CSF leak and 1 (1\%) 


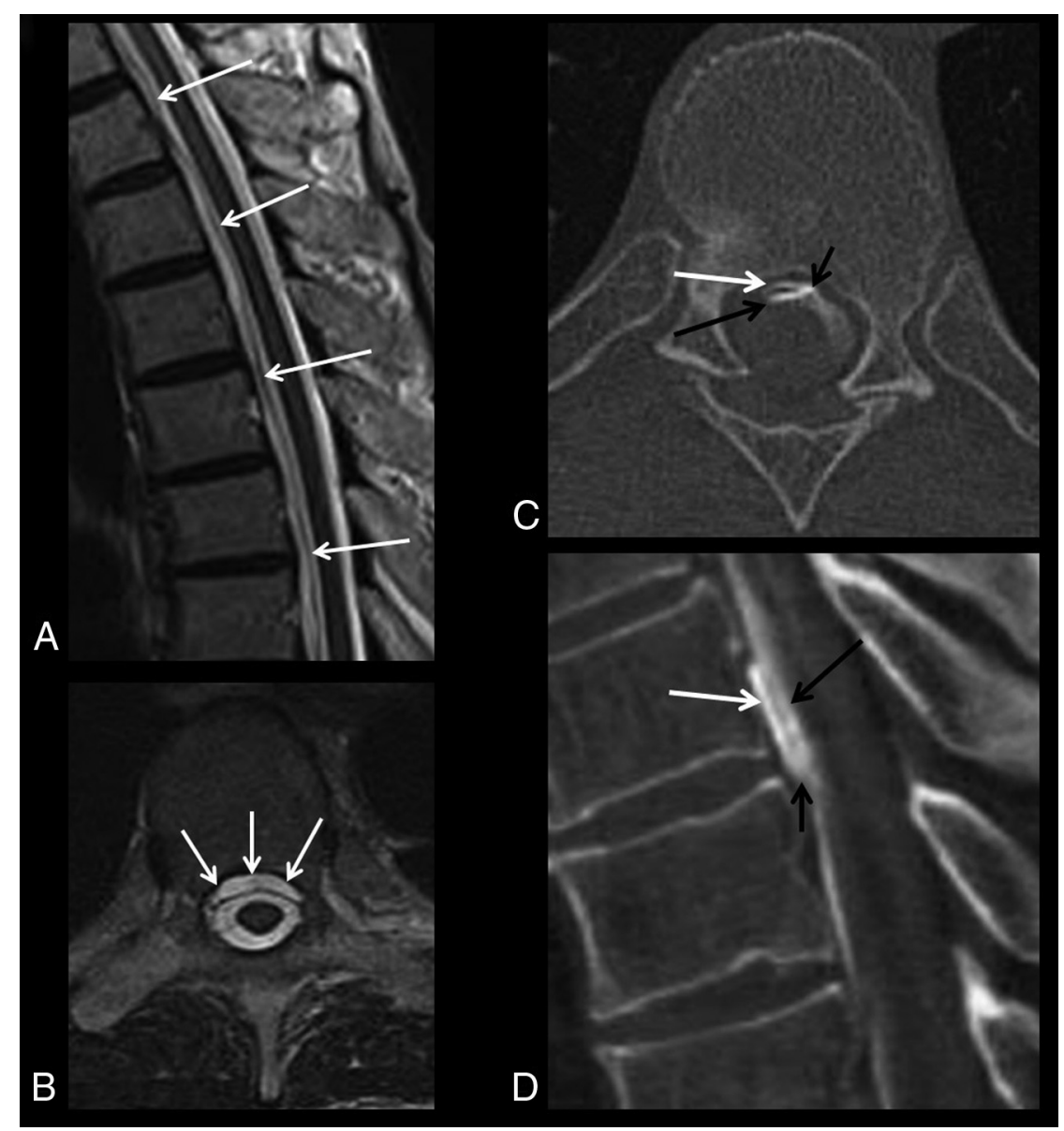

FIG 1. Example of a patient with algorithm compliance and success. Initial spinal MR imaging demonstrates a ventral epidural fluid collection in the midthoracic spine on sagittal (white arrows, $A$ ) and axial (white arrows, $B$ ) T2-weighted images. The patient was appropriately triaged to dynamic CTM, in which a fast CSF leak is identified on initial dynamic axial (C) and sagittal reformatted $(D) C T$ scans. $C$ and $D$, Short black arrows indicate the CSF leak site; white arrows, ventral epidural contrast; and long black arrows, contrast in the ventral thecal sac.

Because extradural fluid could have potentially been present in patients with only partial spinal MR imaging performed, statistical analysis was repeated with exclusion of these patients. This analysis demonstrated similar results, with algorithm breaches resulting in significantly more repeat imaging with dynamic CTM $(P=.013$, 2-tailed Fisher exact test) and a non-statistically significant trend toward more unnecessary initial dynamic CTMs ( $P=.16$, 2-tailed Fisher exact test) than cases in which the algorithm was followed.

\section{DISCUSSION}

While some patients with spontaneous intracranial hypotension have a selflimited course and can be treated with conservative measures, including bedrest, hydration, and caffeine, many do require an invasive therapeutic intervention. ${ }^{3}$ In those patients who do not respond to $\geq 1$ nontargeted epidural blood patch, targeted epidural injections or surgical repair may be required. In these patients, localization of the spinal leak becomes necessary to provide targeted therapy. CTM remains the primary technique for localization of spinal CSF leaks.

This study demonstrates that the imaging algorithm we have adopted for the evaluation of patients with suspected spinal CSF leak, when followed at our institution, results in significantly de-

required repeat imaging with a dynamic CTM for localization of a fast leak.

\section{Algorithm Noncompliance, Breach Types, and Clinical Impact}

The algorithm was not followed in 27 (21\%) of 129 patients. Breaches included no pre-CTM spinal MR imaging in 11 (41\%), performing a conventional CTM when extradural fluid was present on spinal MR imaging in 13 (48\%), and performing an unnecessary dynamic CTM for a slow or absent CSF leak when extradural fluid was absent on spinal MR imaging in 3 (11\%).

Algorithm breaches resulted in $8(30 \%)$ of 27 patients having nonlocalized fast leaks, in 1 (13\%) of 8 due to no pre-CTM MR imaging, and in 7 (87\%) due to performing a conventional CTM when extradural fluid was present on spinal MR imaging. Repeat imaging with dynamic CTM was performed in 4 (15\%) of 27 patients for leak localization.

Overall, algorithm breaches resulted in significantly more repeat imaging with dynamic CTM $(P=.007,2$-tailed Fisher exact test) and a non-statistically significant trend toward more unnecessary initial dynamic CTMs ( $P=.11,2$-tailed Fisher exact test) than cases in which the algorithm was followed. creased repeat dynamic CTM performed for leak localization as opposed to cases in which the algorithm is not followed. In addition, when the algorithm has been followed, rates of repeat dynamic CTM have decreased to $1 \%$ as opposed to an average of $21 \%$ during the 8 years before using the algorithm. Additionally, fewer unnecessary dynamic CTMs for slow or absent CSF leaks have been performed when the algorithm has been followed as opposed to when it has been breached.

Important benefits result from reducing repeat dynamic CTMs. One obvious benefit is a decrease in radiation dose to the patient from only having a single CTM performed rather than a conventional CTM followed by a dynamic CTM. This is particularly significant given the increased awareness and public concern about medical radiation in recent years, with focused effort to keep radiation doses as low as reasonably achievable. ${ }^{4}$ The estimated effective dose for a conventional CT myelogram at our institution is $21.5 \mathrm{mSv}$ compared with $70.6 \mathrm{mSv}$ (range, 21.5$182.9 \mathrm{mSv}$ ) for a dynamic CT myelogram. ${ }^{2}$ Three patients in the current study underwent digital subtraction myelography with an average effective dose of $32.2 \mathrm{mSv}$. Another benefit of reducing repeat dynamic CTM is improved patient care, in that the patient 
has to undergo only a single invasive examination rather than 2, which leads to lower risk, less discomfort, and cost savings for the patient. In the patient population with spontaneous intracranial hypotension, the risk of post-lumbar puncture headache should particularly be minimized, including avoiding unnecessary dural puncture. Although this outcome is speculative, referring clinician and patient satisfaction is presumably heightened because the clinical question is more quickly answered, with the opportunity to enact prompt targeted therapy if necessary. Finally, in a time when health care spending is expected to rise at faster rates in the United States compared with previous years, ${ }^{5}$ judicious use of our resources and elimination of any potentially superfluous examinations are desirable.

Our study did have limitations. Inherent limitations include the retrospective nature of the study and its performance at a single large referral center. Another limitation is that $20 \%$ of patients who underwent pre-CTM spinal MR imaging and whose procedures were considered to have followed the algorithm had only a portion of the spinal canal rather than the entire spinal canal scanned by MR imaging. Extradural fluid could not be entirely excluded in such patients with partial spine MR imaging negative for leak. While it is unclear why these cases occurred, it is postulated that MR imaging examinations were occasionally targeted for clinically suspected symptoms at a specific level. However, the algorithm was successful in all these patients; they either had no extradural fluid detected in the imaged portion of the spine and underwent conventional CTM with slow or no CSF leak identified or had extradural fluid and underwent dynamic CTM with a fast leak identified. In the patients with leaks identified, the spinal segment including the leak level was included on each of the MR imaging examinations. Additionally, repeat statistical analysis with exclusion of patients with only a portion of the spinal canal scanned on MR imaging yielded similar statistically significant results in terms of repeat dynamic CTM and unnecessary initial dynamic CTM between the algorithm-compliant and -noncompliant groups. Ideally, though, each patient would undergo MR imaging of the entire spine to look for extradural fluid before the type of CTM to perform was decided. Timing between MR imaging and CTM was not standardized. An additional limitation of our study is that we could not determine the precise reasons for algorithm breaches, given the retrospective analysis.

Although algorithm success was quite high, several patients had algorithm failure. The reasons for algorithm failure are unclear and may be related to changes in rate/presence of CSF leak between MR imaging and CTM and/or individual patient variability in the ability to absorb CSF from the epidural space. Interventional therapy for the treatment of spontaneous intracranial hypotension, present in 15 patients between spinal MR imaging and CTM, could also potentially have altered the rates of algorithm success and failure. However, most patients who underwent interval treatment had no extradural fluid on MR imaging, were appropriately triaged to conventional CTM, and had no leak identified. Algorithm failure could additionally be secondary to the low sensitivity or specificity of spinal MR imaging for the detection of extradural fluid. No attempt was made to determine the sensitivity, specificity, or interobserver variability of detection of extradural fluid on spinal MR imaging because this study was focused on the effectiveness of the algorithm in routine clinical practice.

Given the high rate of algorithm success, future effort could be dedicated to ensuring compliance with the algorithm. The noncompliance rate of $21 \%$ over the study timeframe could have several causes, one of which may be the lack of awareness or education of referring clinicians in regard to the algorithm. The most common causes of algorithm breach were no pre-CTM spinal MR imaging and performing a conventional CTM when extradural fluid was present. It may be instructive to investigate why these algorithm breaches occurred. A possible solution to these most common breaches would be to require entire spine MR imaging before scheduling CTM for suspected spinal CSF leak and to require a dynamic CTM as the initial CTM if extradural fluid is present on the MR imaging, assuming that there are not contraindications to these examinations. Much of the responsibility for ensuring algorithm compliance also falls on the neuroradiologist performing the CTM, who should be aware of the algorithm and follow it unless there is a compelling reason to proceed otherwise.

\section{CONCLUSIONS}

If targeted therapy is being considered in a patient with a suspected spinal CSF leak, use of the presence or absence of extradural fluid on spinal MR imaging to determine whether a patient should initially undergo dynamic or conventional CTM to localize the leak has been a useful algorithm at our institution, with a relatively high degree of compliance. The algorithm has resulted in a significant reduction in the necessity for repeat CTM with a dynamic technique to localize fast leaks, with a minimal number of unnecessary initial dynamic CTMs performed.

\section{ACKNOWLEDGMENTS}

The authors acknowledge Vicki C. Schmidt and Suson M. Walsh for assistance with the radiology information system data base searches.

\section{REFERENCES}

1. Luetmer PH, Schwartz KM, Eckel LJ, et al. When should I do dynamic CT myelography? Predicting fast spinal CSF leaks in patients with spontaneous intracranial hypotension. AJNR Am J Neuroradiol 2012;33:690-94 CrossRef Medline

2. Thielen KR, Sillery JC, Morris JM, et al. Ultrafast dynamic computed tomography myelography for the precise identification of highflow cerebrospinal fluid leaks caused by spiculated spinal osteophytes. J Neurosurg Spine 2015;22:324-31 CrossRef Medline

3. Mokri B. Spontaneous intracranial hypotension. Curr Neurol Neurosci Rep 2001;1:109-17 CrossRef Medline

4. Huda W. Radiation risks: what is to be done? AJR Am J Roentgenol 2015;204:124-27 CrossRef Medline

5. Sisko AM, Keehan SP, Cuckler GA, et al. National health expenditure projections, 2013-23: faster growth expected with expanded coverage and improving economy. Health Aff (Millwood) 2014;33: 1841-50 CrossRef 